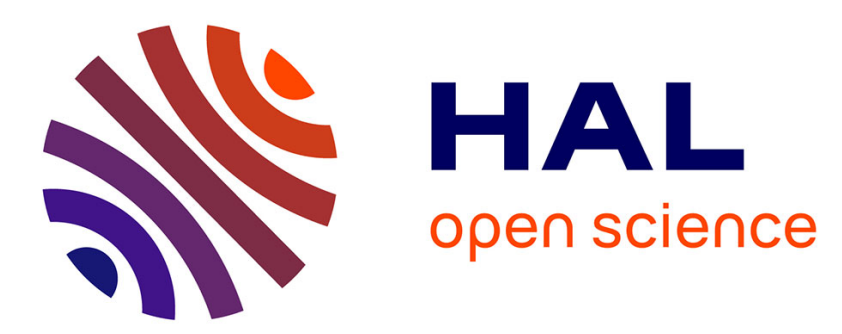

\title{
Tool of the Intelligence Economic: Recognition Function of Reviews Critics. Extraction and linguistic Analysis of sentiments.
}

Grzegorz Dziczkowski, Katarzyna Wegrzyn-Wolska

\section{- To cite this version:}

Grzegorz Dziczkowski, Katarzyna Wegrzyn-Wolska. Tool of the Intelligence Economic: Recognition Function of Reviews Critics. Extraction and linguistic Analysis of sentiments.. 3rd International Conference on Software and Data technologies, ICSOFT 2008, Jul 2008, Porto, Portugal. pp.Pages 218-223. hal-00861380

HAL Id: hal-00861380

https://hal-mines-paristech.archives-ouvertes.fr/hal-00861380

Submitted on 12 Sep 2013

HAL is a multi-disciplinary open access archive for the deposit and dissemination of scientific research documents, whether they are published or not. The documents may come from teaching and research institutions in France or abroad, or from public or private research centers.
L'archive ouverte pluridisciplinaire $\mathbf{H A L}$, est destinée au dépôt et à la diffusion de documents scientifiques de niveau recherche, publiés ou non, émanant des établissements d'enseignement et de recherche français ou étrangers, des laboratoires publics ou privés. 


\title{
TOOL OF THE INTELLIGENCE ECONOMIC: RECOGNITION FUNCTION OF REVIEWS CRITICS. Extraction and linguistic analysis of sentiments
}

\author{
Grzegorz Dziczkowski, Katarzyna Wegrzyn-Wolska \\ Ecole Superieur d'Ingenieurs en Informatique et Genie des Telecommunicatiom (ESIGETEL) \\ 1,Rue de Port de Valvins 77-215 Avon-Fontainebleau Cedex \\ Ecole des Mines de Paris 35, rue Saint-Honore 77305 Fontainebleau \\ grzegorz.dziczkowski@esigetel.fr,katarzyna.wolska@esigetel.fr
}

Keywords: Opinion Mining, Sentiments Analysis, NLP, Recommender System

\begin{abstract}
This paper describes the part of recommender system designed for movies' critics recognition. Such a system allows the automatic collection, evaluation and rating of critics and opinions of the movies. First the system searches and retrieves texts supposed to be movies' reviews from the Internet. Subsequently the system carries out an evaluation and rating of movies' critics. Finally the system automatically associates a numerical mark to each critic. The goal of system is to give the score of critics associated to the users' who wrote them. All of this data are the input to the cognitive engine. Data from our base allow making correspondences which are required for cognitive algorithms to improve advanced recommending functionalities for e-business and e-purchases websites. Our sesystem uses three different methods for classifying opinions from reviews critics. In this paper we describe the part of system which is based on automatically identifying opinions using natural language processing knowledge.
\end{abstract}

\section{INTRODUCTION AND ISSUE}

With the growth of Web, e-commerce has become very popular. A lot of website offer online sales or give the possibilities for rating objects online, for example the movies. While peoples like to check out the recommendations of others users before creating their own opinions those predictions become very useful for the customers. To predict the potential choice Recommender System were created (RS). RS allows people to do the choice without any personal knowledge of alternatives. Algorithms for suggestion are based on the experience and the opinion of other users. It is helpful to find recommendations from people who are familiar with the same problem, who have done their choice in the past, whose perspective we value, or who are recognized experts (Tarveen and Hill, 2001).

RS provides correspondences between the users which have similar profile. A new user has to create his profile. The RS will suggest a new limited choice based on the similar taste of other users. RS proposes the choice to the user which is based on correspondences between the users' tastes. The credibility of the result of RS can not depend on commercial reasons because it could make people distrustful. The efficacy of such system depends of the data's quality and quantity. For this reason presented system furnishes the users' profiles which are necessary for algorithms of cognitive engine. The main goal of the developed system is to collect a huge base of reviews critics and automatically associate marks which express sentiments of the writer. For each critic we associate a new mark and a user profile. The result of this treatment is creation of user's profiles database. Our system is based on statistic and semantic representation of documents. Our work is divided on extraction and filtering the opinion from the text and on assignment the mark to subjective sentences. The extraction and information filtering consists of the identification of quite precise information in a text in the natural language and its representation in a structured form (Panzienza, 1997).

The relative failure of the generic systems comprehension is well-known today. It should however be recalled that these systems resulting from work of automatic treatment of the languages of years 1980 really made it possible to explore this generic approach 
of the comprehension of text.. This is pushing a large numbers of researchers to describe natural languages in the same way as formal languages. Maurice Gross (Gross, 1997) undertook with his team of the LADL (French Laboratory for Linguistics and Information Retrieval) the exhaustive examination of simple sentences of French, in order to have reliable and quantified data on which it would be possible to make rigorous scientific experiments. To exploit the linguistic knowledge an application Unitex was created at LADL (Paumier, 2003). Unitex is an environment of enhancement used to build formalized descriptions to broad coverage of natural languages and apply them as texts of important size in real time. Unitex treat in real time the texts of several mega-bytes for the indexing of morpho-syntactic reasons, the search for set phrases or semi-fixed phrases, and the production of agreements and the statistical study of the results.

Another way to automatically express an opinion from the text is a use of classifier. The statistics methods suppose that descriptions of the objects of the same class are divided by respecting a specific structure of the class. Learning methods based on an example are often used in information's research on a large group of text. Problems consist in constituting a representative corpus of the field which we operate, and to find the rules or to constitute an operational model of this corpus. This model makes the system able to predict the behaviour to adopt when a new candidate arrives to classification. There was a lot of research in classification of reviews to positive and negative like the works of Turney, Littman, Dave, Lawrance, Pang, Lee. Classifiers identify the well-known classes to which belong the objects. The classifiers' performance depends of the model for each class of a base learning (Turney and Littman, 2003), (Wiebe et al., 2004).

\section{LINGUISTIC RESOURCES}

The linguistic resource to achieve the information retrieval and extraction are as follows: dictionaries, networks of the recursive transitions (local grammar, tables of lexicon-grammar.

The digital dictionaries employed by Unitex use formalism of DELA . Numeric dictionaries describe both the simple words and the complex words of a language. Dictionaries associate the word with a lemma and a series of grammatical, semantical and inflexional codes.

Grammar is a representation of linguistic phenomena by recursive transitions (RTN), formalism close to that of the finite state automaton. Many studies have highlighted the adequacy of automats on linguistic problems. A transducer with a finite number of states is a graph which represents a whole of entry sequences, and associates sequences produced as an output. Generally a grammar represents sequences of words and produces linguistic information like the information on the syntactic structure.

A local grammar (Kamp, 1981) is an automaton representation of the linguistic structures witch is difficult to formalize in lexicon-grammar tables or numeric dictionaries. The local grammars, represented in the forms of graphs, describe elements which concern the same syntactic or semantic field. The linguistic descriptions grouped together in the form of local grammars are used for a large variety of automatic processes applied to the text. Thus various methods of lexical clarification were developed to implement grammatical constraints described before using this type of graph.

The corpora of text are represented by automats, in which each state corresponds to a lexical analysis. The linguistic phenomena are represented by local grammar, and are then translated into finite state automaton in order to be easily confronted with the corpora of text.

Tables of lexicon-grammar are matrixes that outline the properties of all the simple verbs which are described by syntactic properties. Each word having almost unique behaviour, the tables give the grammar of each element of the lexicon, which is why they are called lexicon-grammar tables. With Unitex we can build grammar from such tables. The lexicongrammar is a systematic description of the syntactic and semantic properties of the syntactic factors that is predicative verbs, nouns and adjectives. It is organized in groups of tables, which are associated with the syntactic category like full verbs, verbs supports, names, etc... A table corresponds to a particular syntactic construction and gathers all the words entering this construction. Currently lexicon-grammar is especially developed for the verbs and the predicative phrases (Tarveen and Hill, 2001) (Turney and Littman, 2003).

\section{OVERVIEW OF GENERAL APPROACH}

Our system has modular architecture. The principle tasks are: collecting the reviews from Internet, checking if the text found is a review, assigning a mark to the reviews and presentation of results. This paper is focused on the marking critic's module and more precisely of linguistic method of classifying the re- 
views. We developed three different methods for assigning a mark to the reviews. These methods are based on different approach of corpus classification. For each method we developed a classifier which separately assigna a mark. At the end we obtained three marks for one review which can be different. We use another classifier which will assign the final mark to the reviews based only on three marks get before from classifiers (Dziczkowski and Wegrzyn-Wolska, 2007a), (Dziczkowski and Wegrzyn-Wolska, 2007b).

The process of assignment of the mark into the critic is shown on figure 1.

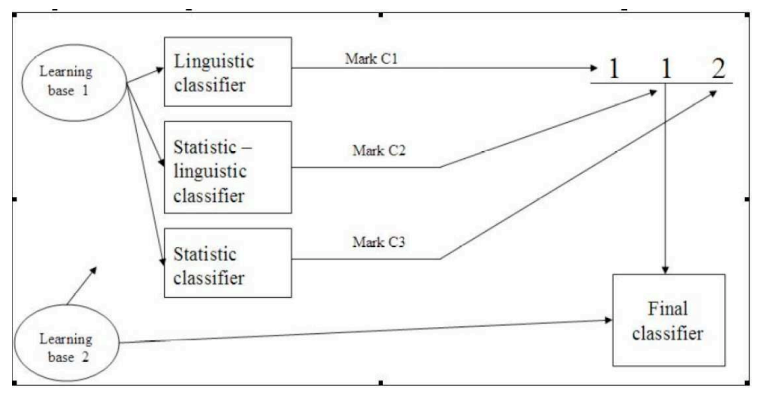

Figure 1: The process of mark assignment

For marking reviews we use three different approaches which are as follows:

- Linguistic classifier: For each sentence of reviews we assign a rule of grammar that expresses intensity of opinion.

- Statistic-linguistic classifier: Statistic researches on linguistic data for determine behaviour of reviews which have the same mark. The futures are for example: characteristic words, sentence length, corpus width, detection of negation, characteristics expressions, special and special punctuation. For entire corpus of reviews we calculate the distance of the characteristics of new reviews to the characteristics of the groups.

- Statistic classifier: Statistic research based on classifier of Bayes which is a categorizer of the probabilistic type founded on the theorem of Bayes.

The work presented in this paper is foccused on the linguistic knowledge using linguistic resource described in section 2 (Cover, 1991), (Dave et al., 2000), (Pang and Lee, 2004), (Wang et al., 2003).

\section{LINGUISTIC CLASSIFIER}

To perform the critics marking we have to get a group of characteristic already evaluated - a learn- ing base. On different website we can find film critics with the mark assigned (e.g. IMDB, Amazon). We used those data (critics, users, marks) to create our learning base. We use the scale of marking from 1 to 5 . We regrouped all the critics by their mark. So we have obtained 5 different groups of film's critics: a group of critics with score 1, 2 ... 5. For each group we build a grammar. Grammar is based on learning base, which contain about 2000 sentence for each mark' group (Dziczkowski and Wegrzyn-Wolska, 2007b).

For this part we use a linguistic treatment which require lexicons and specialized grammar. The development of such resources is a long and tiresome task, which generally requires an expertise on the field approached and knowledge in data-processing linguistics like techniques of filtering, categorization of documents and extraction of information. Comprehension is seen as a transduction which transforms a linear structure, i.e. text (the linear structure) is transformed into an intermediate logico-conceptual representation, which is then used to make conclusions. The semantic analysis aims to produce a structure representing as accurately as possible, a unit of the sentence, with its meanings and its complexity; then it has to integrate all structures into a single textual structure. At the end, we obtain a logico-conceptual representation of the text (Altai, 1992), (Kamp, 1981), (Alshawi, 1992). Semanticoconceptual structures can be more or less broad, rich and complex and more or less ambiguous (Dziczkowski and Wegrzyn-Wolska, 2007a).

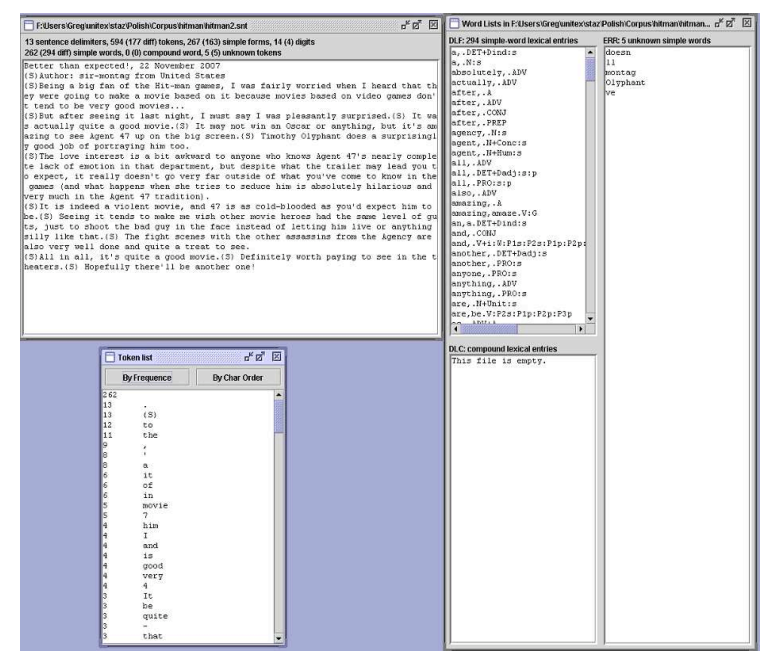

Figure 2: Linguistic resource : dictionaries.

This part of system was developed with Unitex application, the example of linguistic resource used is 
shown on figure 2, figure 3 and figure 4 . We use a linguistic analyser Unitex to pre-treatment, to lemmatise the words, to add synonyms, to detect negation, to add semantic classes to the words and at least to build complex local grammars. Semantic classes are associated to the word and show polarity and intensity of the word. For associate semantic classes to the words we were based on subjective word dictionary General Inquirer Dictionary.

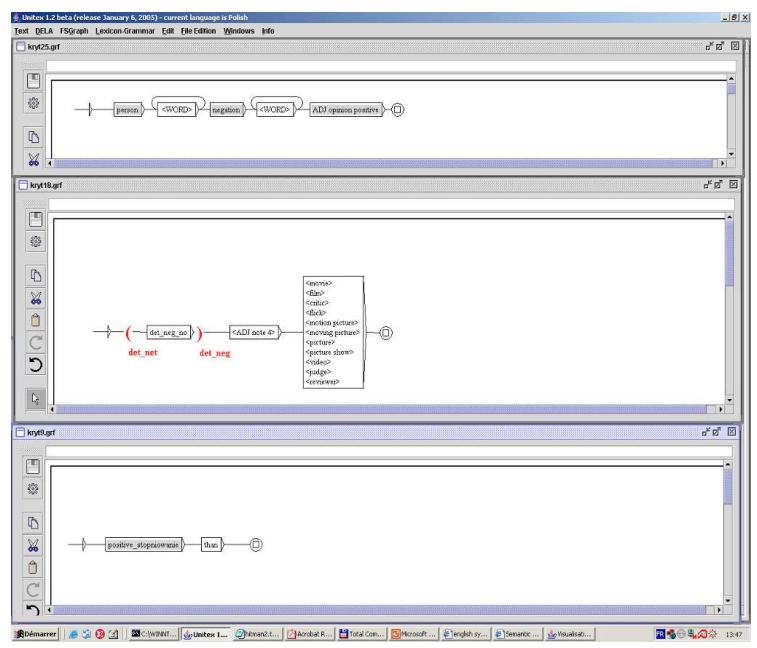

Figure 3: Linguistic resource : local grammar.

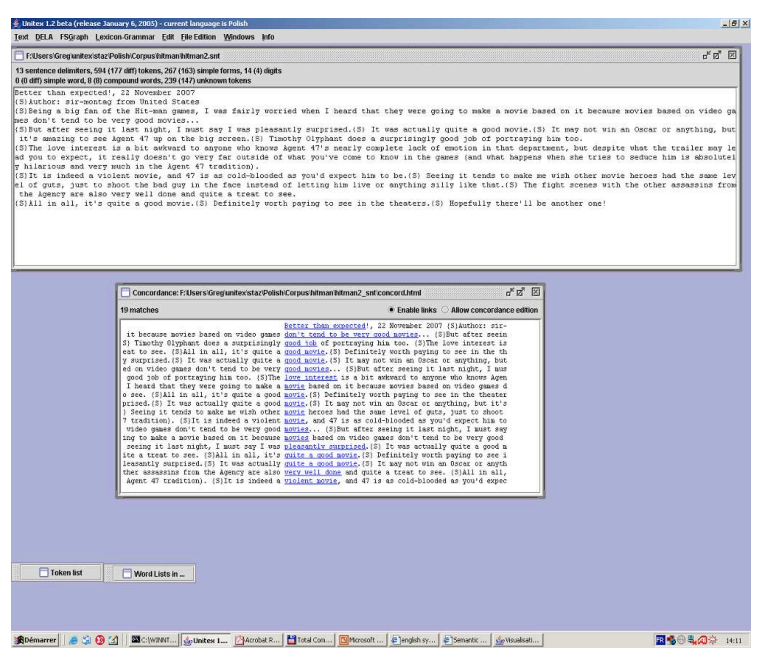

Figure 4: Linguistic resource : results.

The General Inquirer is a mapping tool. It maps each text file with counts on dictionary-supplied categories. It combines the "Harvard IV-4" dictionary content-analysis categories, the "Lasswell" dictionary content-analysis categories, and five categories based on the social cognition work of Semin and Fiedler, making for 182 categories in all. Each category is a list of words and word senses. Unlike some artificial intelligence programs that can be applied to texts within limited topic domains, the General Inquirer simply maps text according to categories and does not search after meaning. General Inquirer mappings have proven to supply useful information about a wide variety of texts. But it remains up to the researchers, not the computer, to create knowledge and insight from this mapped information, usually situating it in the context of additional information about the texts' origins. It contains 1,915 words of positive outlook, 2,291 words of negative outlook. Below on figure 5 is an example of General Inquire Dictionary.

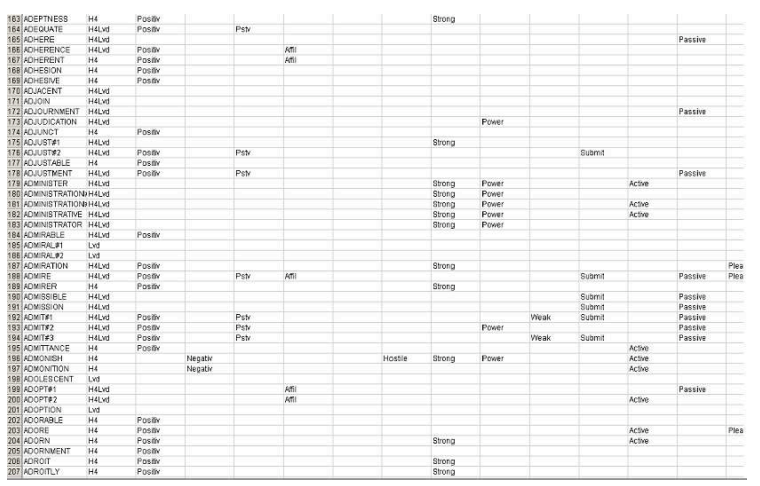

Figure 5: General Inquire Dictionary

The main purpose of linguistic classifier is the assigning of the mark in harmony with sentiments contained in the review. The assignment of mark is carrying on sentence by sentence. In order to create rules of grammar for each mark (in our case the mark from 1 to 5) the study of reviews from the learning base was perform. In this way 5 grammars was created one for each mark. Each grammar contains a lot of rules - local grammars. For each grammar more than 30 local grammars was created. In order to assign the mark to the new opinion research is performed sentence by sentence in order to find the rule corresponding to the examined sentence. At the end of this treatment we obtained selected sentences of new reviews with corresponding rules. To obtain the final mark we calculate the average of marks corresponding to main grammars.

The constructions of local grammars were done in manual way by analysing of reviews sentences with the same mark associated. The local grammar can not be to much general cause it makes the research too much ambiguous. If the local grammars is too much complex the application is doubtful. The local gram- 
mars were created for detection the polarity and intensity of opinion for one sentence. Other classifiers used in our system perform the statistic classification. In this classifier we just take care of form of local grammars. Other more statistic futures like typical words, typical expression, size of sentence the frequency of characteristic word repetition, the number of punctuation marks are not taken in account. Of course the typical words are in dictionaries with semantic classes and in local grammars, but the grammar must exist for linguistic treatment. In figure 6 there we show an example of local grammar.

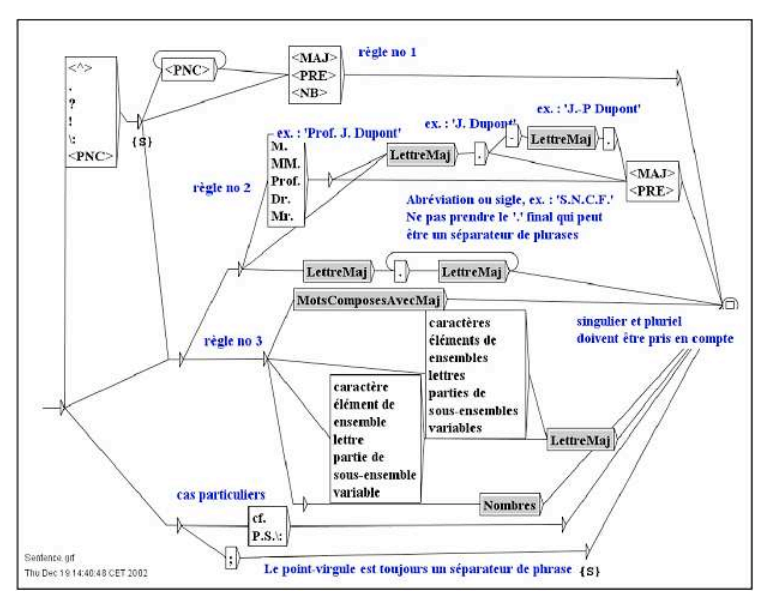

Figure 6: Example of local grammar

The creation of local grammar is a timeconsuming task. And it's difficult to explain in scientific way if the local grammars couldn't be done better or on which complex level we should stop. The grammars used in our system were accordant in empiric way. We start to create local grammars. Then we added the level of complicity of local grammars and so on. For each level we effected tests and calculated F-score. The final result of our rules of grammars is chosen to provide the best F-score. Unfortunately we can not be sure that our choice is the most coherent. We took into consideration that each classifier presented in our system should have its own futures. In spite of all it's important to notify that linguistic classifier gives the best results.

\section{RESULTS}

We carried out tests of presented linguistic classifier for all groups of mark. The corpus of movie reviews used in test contains 2264 sentences for a mark equal to 5,1957 sentences for 4,1308 sentences for 3,1925 sentences for 2 , and 1835 sentences for 1 . The results are shown in Table 1.

Table 1: Experimental results

\begin{tabular}{|c||c|c|c|}
\hline & Precision & Recall & F-score \\
\hline Class 5* & $72.4 \%$ & $83.4 \%$ & $76.5 \%$ \\
Class 4* & $70.8 \%$ & $82.4 \%$ & $76.1 \%$ \\
Class 3* & $67.8 \%$ & $71.6 \%$ & $69.6 \%$ \\
Class 2* & $62.5 \%$ & $55.9 \%$ & $59 \%$ \\
Class 1* & $76.3 \%$ & $84.2 \%$ & $80.1 \%$ \\
\hline
\end{tabular}

We can see that the better results were obtained for the extreme opinion - for the movies reviews with a mark equal to 1 or 5 . Results seem to be logical because extreme emotions are strongest, so it is easiest to automatically mark and to judge them all. Moreover extreme reviews are most often longest so it supports the correct assessment. In spite of these improvements we made, we are still far from the ideal case. According to our test results and since it is necessary to start from the principle that more complex and complicated grammars are needed, we noticed that the linguistic classifier gives better results that statistic or statistic-linguistic classifier.

\section{CONCLUSIONS}

Presented system caries out a collection of movies critics and automatically assign a mark to each critic. This system is a support of RS. The goal of our work is to automate the whole system, particularly to improve the estimation of individual user's critics. The system allows an automatically assignment of a mark; however to increase the research on other fields it will be necessary to create a linguistic base and a new analyze of the different elements of the group's behaviour.

We focused ourselves on the automatic search task of information in a corpus, more precisely on the linguistic analyse of sentiments. Our study was made on the application "Unitex" since it's the tool that makes it possible to carry out a major search by using grammars, tables of lexicon-grammar and dictionaries. Our objective was to prepare the data and creation of complex local grammars.

We succeeded in the creation and the integration of linguistic classier. This method made possible to automatically assign a mark to the sentiments in movies reviews. The adjustment of the linguistic resources like the creation of the complex local grammars or the adaptation of the dictionaries was an im- 
portant part of our work to improve the linguistic classifier. We obtained satisfying results, but it is necessary to specify that there remain several points to be improved. The solutions from the automatic information retrieval presented in this report give an image of the complexity of this field and highlight the need for making improvements and especially for opening several doors in the domain of research.

\section{REFERENCES}

Alshawi, H. (1992). The core language Engine. MIT Press.

Altai, H. (1992). The core language engine. In ACL-MIT Press Series in Natural language Processing. MIT Press.

Cover, T. (1991). Elements of Information Theory. John Wiley.

Dave, K., Lawrance, S., and Pennock, D. (2000). Opinion extraction with hmm structures learned by stochastic optimization. AAAI.

Dziczkowski, G. and Wegrzyn-Wolska, K. (2007a). Graph based system purpose - built for automatic retrieval and extraction of the electronics data. In Internet and Multimedia Systems and Applications. ACTA Press.

Dziczkowski, G. and Wegrzyn-Wolska, K. (2007b). Rcss rating critics support system purpose built for movies recommendation. In Advances in Intelligent Web Mastering. Springer.

Gross, M. (1997). The construction of local grammars. In Finite-State Language Processing. MIT Press.

Kamp, H. (1981). Evenements representations discursives et reference temporelle. In Langages $n b 64$.

Pang, B. and Lee, L. (2004). Sentimental education: Sentiment analysis using subjectivity summarization based on minimum cuts. In $A C L$.

Panzienza, M. (1997). Information extraction (a multidisciplinary approach to an emerging information technology). Springer Verlag (Lecture Notes in Computer Science), Heidelberg,

Paumier, S. (2003). De La reconnaissance de formes linquistique a l'analyse syntaxique. These, Marne-laValee,.

Tarveen, L. and Hill, W. (2001). Beyond recommender systems: helping people help each other. In $\mathrm{HCI}$ in the millennium. Addison-Wesley.

Turney, P. and Littman, M. (2003). Measuring praise and criticism: Inference of semantic orientation from association. In ACM Transactionon Information Systems. TOIS.

Wang, Y., Hodges, J., and Tang, B. (2003). Classification of web documents using a naive baves method. IEEE.

Wiebe, J., Wilson, T., Bruce, R., Bell, M., and Martin, M. (2004). Learning subjective language. computational linguistics. 